\title{
Toxoplasma infection during pregnancy. Counselling and screening challenges
}

\author{
Laura Dumitrasi', Corina Gica', Radu Botezatu1,2, Nicolae Gica, ${ }^{1,2}$, \\ Anca Marina Ciobanu ${ }^{1,2}$, Gheorghe Peltecu ${ }^{1,2}$, Anca Maria Panaitescu ${ }^{1,2}$ \\ ${ }^{1}$ Filantropia Clinical Hospital, Bucharest, Romania \\ 2"Carol Davila" University of Medicine and Pharmacy, Bucharest, Romania
}

\begin{abstract}
Toxoplasmosis is the most common cause of congenital infection, causing long term ocular and neurological lesions with relapses during childhood and adolescence. The risk of congenital infection is highest after primary maternal infection with advancing maternal gestation. Only a few european countries recommend a routine prenatal screening although therapeutic options are the same.

Primary prevention measures regarding food hygiene and secondary prevention through serological testing are the most important ways to prevent and detect toxoplasmosis during pregnancy. Counselling is challenging since there is a need for reference centers of diagnosis.

In Romania there is a routine clinical practice to offer screening through the TORCH panel in the first trimester of pregnancy.

This review summarizes the current methods of diagnosis and management of toxoplasmosis in pregnancy.
\end{abstract}

Keywords: Toxoplasma gondii, pregnancy, diagnosis, treatment

\section{INTRODUCTION}

Toxoplasma gondii (T. gondii) infection is one of the major causes of congenital infection leading to visual and neurological deterioration of the fetus or death. The estimated incidence of congenital toxoplasmosis in Europe is about 1-10/10,000 newborn, though it is hard to predict the incidence and severity as this depends on the trimester in which the infection affects the mother (1) and the earlier the infection the more severe sequelae in offspring are seen (2).

T. gondii is a protozoan parasite existing in different forms: the invasive form called trophozoite and the latent form called oocyst or bradyzoite (3). Transmission of parasites usually occurs without the knowledge of the patient and is not related to direct exposure to a cat, especially if it is an indoor cat fed with only cooked or canned food, but by ingestion of vegetables, undercooked meat or water contaminated with oocysts (4).
There is only one species of Toxoplasma, with a great genetic diversity (5) having 3 genotypes. In has been suggested that the clinical spectrum of congenital toxoplasmosis (CT) depends on the strain predominantly affecting an area, with type 2 affecting Europe and North America and non-type 2 strains, which are more virulent, in South America, associated with severe visual impairment in children and preterm birth (6).

Congenital infection develops through transplacental transmission of $T$. gondii, as it can colonize the placenta during the acute stage of infection, continuing as a source of infection if it is left untreated (7). Congenital toxoplasmosis (CT) occurs most frequently after maternal acute primary infection during pregnancy or within 3 months prior to conception. Less commonly, $\mathrm{CT}$ can occur through reactivation of the infection in an immunocompromised pregnant woman or after reinfection with a new, more virulent strain (6). 
The risk of maternal-fetal transmission of infection is about $25-30 \%$, but the rate of vertical transmission is directly related to gestational age and it increases from $10 \%$ to $15 \%$ in the first trimester, to $25 \%$ in the 2 nd trimester, and over $60 \%$ in the 3rd trimester. If vertical transmission occurs, the risk of symptomatic infection at birth decreases with advancing gestational age at the time of infection, from $60 \%$ in the first trimester to $10 \%$ in the third trimester (8). Most infected infants $(90 \%)$ do not have clinical signs of infection at birth, while $10 \%$ of neonates will present chorioretinitis, intracranial calcification or hydrocephalus. In some cases of asymptomatic neonates, sequelae such as chorioretinitis, hearing loss or neurodevelopmental delay can develop later in life $(7,9)$.

Preventive precautions for the immune negative pregnant women are of great importance. Standardised therapy regimes have been developed depending on the gestational week and the earlier materno-fetal therapy is initiated, from the stage of suspected infection, the better the prognosis of the neonate is (10).

Diagnosis and management of toxoplasmosis during pregnancy are undertaken by a multidisciplinary team which involves obstetrician, fetal medicine specialist, parasitologist, ophthalmologist, paediatrician or neurologist (11).

Every country has developed different public health strategies to prevent congenital toxoplasmosis depending on the level of prevalence, type of strain (virulent versus less virulent) and incidence of the disease as well as on the cost of screening $(2,6)$.

Screening for maternal toxoplasmosis in Romania has become a routine practice, though is not part of a prenatal programme. It starts with the acquisition of immunoglobuline $\mathrm{M}$ (IgM) and immunolgobuline $\mathrm{G}$ (IgG) antibodies through the TORCH panel at the first prenatal visit usually in the first trimester, followed by Ig $\mathrm{G}$ avidity measurements in cases of Ig M and Ig G positivity. Treatment is usually prescribed by the parasitologist before specific fetal tests of confirmation are done, who also scheduels further serological testing and changes the treatment, if necessary, when the fetal infection is discovered. There is a routine ultrasound follow-up monthly or twice a month of the affected women by the maternal fetal medicine specialist who decides whether or not perform an amniocentesis for confirmation of fetal infection. If a pregnant woman is tested negative for toxoplasmosis in the first trimester, some practitioners recommend further toxoplasmosis antibodies measurement, at every three months, to exclude a latter infection. After birth, neonatologists recommend neurological and ocular follow-up to exclude and treat affected children. Management of toxoplasmosis during pregnancy in Romania usually implies a team formed by an obstetrician, parasitologist and fetal medicine specialist.

American College of Obstetricians and Gynecologists (ACOG) and Royal College of Obstetricians and Gynecologists (RCOG) do not recommend routine screening for toxoplasmosis in pregnancy. Although screening is not recommended, pregnant women should be advised regarding primary prevention measures (12). Prenatal programmes exist in several countries with differences in the testing scheduel and costs. Despite these efforts, compliance affects the effectiveness of screening.

A decrease in the severity of toxoplasmosis has been observed in some European countries using routine antepartum screening (6).

France, Italy regional, Germany and Denmark are countries with a specific surveillance for diagnosis of congenital toxoplasmosis (11).

\section{PRIMARY INFECTION IN PREGNANCY}

\section{Maternal signs and symptoms}

Asymptomatic infection predominates (over 80\% of cases), however an acute systemic infection, developing 5 to 23 days after exposure to the protozoa can appear with an incubation period of 5 to 18 days (17).

Mild symptoms like fever lasting 2-3 days, chills, sweats, headaches, myalgias, pharyngitis, hepatosplenomegaly or a diffuse non-pruritic maculopapular rash can occur. Lymphadenopathy that is bilateral, symmetrical, nonfluctuant and nontender cervical adenopathy, sometimes persisting for weeks, is common in acute infection (18). Chorioretinitis with visual impairment during acute maternal infection is rare (19).

Diagnosis of maternal toxoplasmosis

Any suspicion of toxoplasmosis in a pregnant woman should be confirmed through measurement of IgG and IgM within the initial evaluation (3). Testing earlier in pregnancy (1st trimester in particular) has proved to be more clinically helpful than testing in the 2nd trimester (19).

In acute infection, IgM antibodies appear within 7 days and reach a maximum individual titer. The rate 
TABLE 1. Screening/non-screening programmes all over the world

\begin{tabular}{|c|c|}
\hline France & 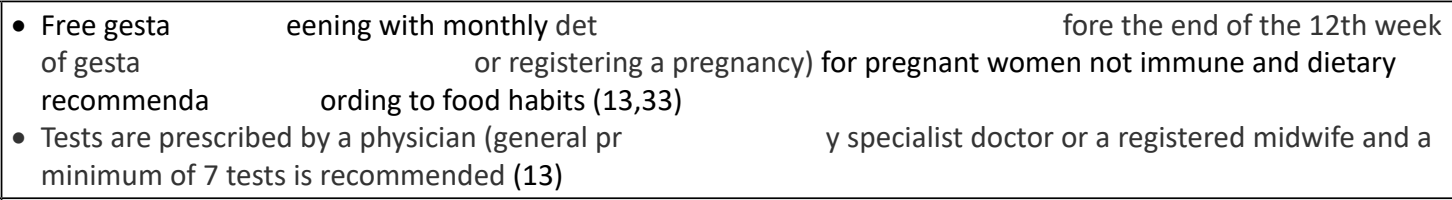 \\
\hline Austria & 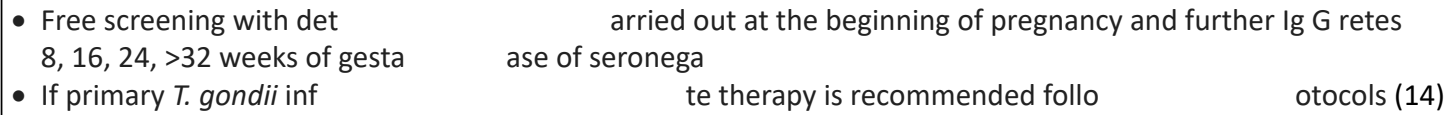 \\
\hline Italy (regional) & $\begin{array}{l}\text { - Serological prenatal screening is done by the 13th week of pregnancy; if seronega e, } 1 \text { repeat tests every } 30-40 \\
\text { days un ery for a total of 5-7 screenings (1) }\end{array}$ \\
\hline $\begin{array}{l}\text { Bulgaria, Cyprus } \\
\text { - tes or }\end{array}$ & $\begin{array}{l}\text { Czech Republic, England and Wales, Estonia, Ireland, Latvia, Lithuania, Malta, Poland, Scotland and Slovakia } \\
\text { s\$ } \$ \text { mptoma ongenital toxoplasmosis (11) }\end{array}$ \\
\hline $\begin{array}{l}\text { Morocco, Colon } \\
\text { - regional high } \\
\text { - maternal an }\end{array}$ & $\begin{array}{l}\text { idence of congenital toxoplasmosis } \\
t \text { t } \quad t \text { the } 1 \text { st prenatal visit in } I\end{array}$ \\
\hline Germany & tlinien) recommend serological tes \\
\hline USA & $\begin{array}{l}\text { - absence of a na tep国rtum screening; however some physicians pr } \\
\text { - serologic screening in pregnancy only in suspected maternal or fetal inf }\end{array}$ \\
\hline Switzerland & $\begin{array}{l}\text { - Absence of mandatory gesta eening } \\
\text { - Performing cord blood screening for congenital toxoplasmosis since } 1982 \text { with declining seroprevalence over } \\
\text { years (34) }\end{array}$ \\
\hline
\end{tabular}

of Ig $\mathrm{M}$ decline varies among patients, taking up to several months or years (4). Toxoplasma-specific IgG antibodies appear within 14 days of primary infection, with a maximum titer at 8 weeks and persisting throughout life in different titers. A high level of IgG should not always suspect a recent infection (3). In cases of equivocal results or suspected seroconversion, it is useful to obtain serum specimens 3-4 weeks apart and pay attention also to the rise of $\operatorname{IgG}$ titer; usually a 4-fold rise is seen in seroconversion (4). Reference laboratories are important for aditional testing, especially for patients with positive or equivocal IgM antibody test results (19).

Negative IgG and IgM defines no serologic evidence of prior infection, but the risk for acute infection and CT still exists throughout pregnancy and follow-up tests can detect seroconversion (positive $\operatorname{IgM}$ and $\operatorname{IgG})$. In this stage, counseling should focus on the primary preventive measures (19).

Positive IgG and negative IgM under 18 weeks gestation should exclude CT in immunocompetent patients. After 18 weeks of gestation, obtaining previous serological tests can prove helpful in defining the time of infection (19).

Negative IgG and positive IgM recommend repeating tests in 1-3 weeks. With a positive IgM and IgG of the new tests, seroconversion should be suspected, begining treatment according to protocol. It is important to start ultrasound follow-up and consider amniotic fluid PCR after 18 weeks gestation (19).

If both IgG and IgM are positive, confirmatory testing, preferably to a reference laboratory, is needed such as Ig A, Ig E, Ig G avidity. IgA antibodies are used more in diagnosis of the infection in fetus and newborn rather than in the adult (3). IgE antibodies have a shorter seropositivity than $\operatorname{IgM}$ and $\operatorname{IgA}$ and are detected in cases of acute adult infection, congenital infection in children and chorioretinitis (3).

IgG avidity measurements should not be used alone in establishing the time of infection, especially in low avidity Ig $G$ result. In case of a high-avidity Ig $\mathrm{G}$ test, despite high Ig M titer, within 16 weeks of gestation, one should consider an infection 12-16 weeks earlier and a minor transmission rate $(8,19)$. It is important to provide as much clinical information as necessary about the mother and infant in order to have a better interpretation of serological tests and specific recommendations (19).

\section{When to suspect congenital toxoplasmosis?}

In the absence of maternal serologic screening there are sonographic nonspecific signs that may lead to the suspicion of CT. However, one must perform a maternal serology for $T$. gondii to confirm infection and recommend further invasive fetal diagnosis. 
TABLE 2. Fetal ultrasound findings reported in the literature to be associated with CT (6)

\begin{tabular}{|c|c|c|}
\hline $\begin{array}{l}\text { Central nervous } \\
\text { system (CNS) signs }\end{array}$ & Extracerebral signs & General signs \\
\hline $\begin{array}{l}\text { 1. Ventricular } \\
\text { dila } \\
\text { 2. Hydrocephalus } \\
\text { 3. Intracranial }\end{array}$ & $\begin{array}{l}\text { 1. Ascites } \\
\text { 2. Echogenic bowel } \\
\text { 3. Hepatosplenomegaly } \\
\text { 4. Intrahepa } \\
\text { c a ? ? }\end{array}$ & $\begin{array}{l}\text { 1. Fetal } \\
\text { demise } \\
\text { 2. Intrauterine } \\
\text { growth } \\
\text { res }\end{array}$ \\
\hline c a ? 回 & $\begin{array}{l}\text { 5. Placental } \\
\text { hyper } \\
\text { placenta increased } \\
\text { thickness } \\
\text { 6. Pericardial and/or } \\
\text { pleural e }\end{array}$ & $\begin{array}{l}\text { 3. Hydrops } \\
\text { fetalis }\end{array}$ \\
\hline
\end{tabular}

Ultrasonographic signs and positive maternal screening increase the suspicion of CT. In this case, amniocentesis with PCR for T. gondii DNA in amniotic fluid is recommended beyond 18 weeks of gestation for prenatal diagnosis of fetal infection. A delay of 2 weeks after serological seroconversion or 4 weeks after maternal primary infection achieves a better diagnosis (20). Cordocentesis is no longer recommended due to its higher risk to the fetus (3), making amniocentesis the standard diagnostic test with a sensitivity of $90 \%$ and a specificity of $100 \%$ if performed after 18 weeks. Amniocentesis should be avoided in HIV infected women and under 18 weeks of pregnancy (4).

In case of negative amniocentesis, ultrasound follow-up every month should continue to detect delayed abnormalities that could have a rapid onset in case of virulent strains of $T$. gondii. Counseling the parents about this risk is recommended until delivery. If ultrasonographic abnormalities are detected and therapeutic abortion is considered, a necropsy to confirm toxoplasmic etiology is required (21).

In case of positive amniocentesis, ultrasound follow-up is increased at a frequency of twice a month, with the possibility of fetal brain MRI at 30-32 weeks of gestation for better cerebral evaluation (5). Testing the placenta for $T$. gondii has not proved useful ( $25 \%$ sensitivity) (22).

Treatment regimens for toxoplasmosis depend on the gestational age at the time of diagnosis. For a suspected or confirmed infection under 18 weeks gestation, Spiramycin is recommended, followed by amniotic fluid PCR after 18 weeks gestation and ultrasound follow-up. A negative PCR and normal ultrasound findings recommend continuing Spiramycin until delivery with aditional monthly ultrasound follow-up.
A positive PCR or abnormal ultrasound findings recommend switching treatment to Pyrimethamine plus Sulfadiazine plus Folinic acid, until delivery (19).

After 18 weeks gestation in case of suspected or confirmed infection there is a recommendation for Pyrimethamine plus Sulfadiazine and Folinic acid with fetal ultrasound and amniotic fluid PCR. The initial 3 drugs treatment could be continued until delivery regardless of the PCR and ultrasound results or could be switched to Spiramycine only if both PCR and ultrasound are negative (19). The gestational age for beginning the 3 drugs regimen could be even lower than 18 weeks as Toxogest trial sugests, being the only randomised trial that tested the efficacy of pyrimethamine-sulfadiazine vs. spiramycin on reducing maternal-fetal transmission. The results of the study support prophylactic treatment with Pyrimethamine and Sulfadiazine in all cases of maternal seroconversions after 14 weeks of gestation to reduce the rate of vertical transmission. The treatment should be initiated within 3 weeks after seroconversion $(30,31)$. Initiating treatment as soon as seroconversion is discovered, even if the mother is asymptomatic, reduces the prenatal cerebral findings, eye disease and hydrocephalus in fetuses and later in life $(30,32)$.

Timing or route of delivery should not be affected by CT (2) as prevention of transplacental transmission does not seem to be improved by prompt delivery (cesarean or induction of labour) (23). Breast feeding and immunization programs are not contraindicated (16).

\section{NEONATAL DIAGNOSIS}

In case of confirmed or suspected primary maternal infection during pregnancy, regardless of the amniocentesis result or ultrasound findings, all neonates should be tested for CT on day 2 or 3 of life (5) and repeating tests after 1 week (24).

Diagnosis or exclusion of CT is best to be done in consultation with toxoplasmosis reference laboratories. The presence of IgG only do not define CT (5) and have been detected until 12 months of age due to physiological passage of IgG through the placenta from the mother to the fetus (25). The gold standard diagnostic for $\mathrm{CT}$ is persistence of positive Toxoplasma IgG antibodies beyond 12 months of age. Positive diagnosis is also defined by positive Toxoplasma IgG antibodies and positive Toxoplasma IgM antibodies 
and/or positive Toxoplasma IgA antibodies or positive PCR from amniotic fluid, peripheral blood, cerebrospinal fluid (CSF), urine, or other body fluids. Congenital toxoplasmosis should not be excluded if the mother had clinical manifestations of toxoplasmosis, with serological confirmation, but the neonate has only IgG antibodies present. It is recommended to repeat clinical and serum evaluation of $\mathrm{IgG}$ antibodies every 4 to 6 weeks after birth until complete disappearance in order to eliminate any suspicion of CT. Further clinical evaluation consists in meticulous physical, neurological and ophthalmologic examination (with retina specialist), as well as brainstem auditory evoked responses. Imaging evaluation with computed tomography of the head (or head ultrasonography) and abdominal ultrasonography have proved helpful (6).

Postnatal treatment for infants with CT follows certain protocols and dosages and includes pyrimethamine, sulfadiazine and folinic acid with clinical, ophtalmological and serological follow-up. Clinical and ophthalmological surveillance is recommended: every 3 months during the 2nd year of life, every 6 months during the 3rd year of life and one every year throughout life (5) as visual or hearing impairment with learning disabilities can manifest later (26).

Retinal infection with $T$. gondii is one of the most common cause of infectious posterior uveitis, leading to irreversible damage, large scotomas or blindness (15). The occurence of chorioretinitis in offspring depends on whether the mother received treatment during pregnancy ( $25 \%$ with visual impairment) or not ( $72 \%$ with chorioretinitis) (26). Ocular toxoplasmosis manifests as bilateral focal necrotizing retinitis (27). It is important to perform the ophtalmological surveillance once a year especially in childhood, adolescence and pregnancy because of its uncertain recurrence (5).

The variety of CNS involvement is represented by internal obstructive hydrocephalus (which can appear after birth and progress necessitating neurosurgical intervention), seizures, spinal or bulbar involvement manifested by paralysis of the extremities, difficulty in swallowing, cerebral calcifications. Neurosurgical shunt placement usually improves the intelligence quotient (IQ) within normal values (27). Around 5-9\% of children with CT present cerebral calcifications which are difficult to associate with cerebral hyperecogenic foci in the prenatal period and further neurological development. The presence of calcifications represents a risk factor for retinochoroiditis developing during childhood (28). The cognitive function tests are affected mainly through ophtalmologic rather than neurologic involvement (29).

\section{CONCLUSIONS}

Despite improvements in treating toxoplasmosis during pregnancy, diagnosis and management of infection are a challenging task.

There is a general debate on universal screening in the 1st trimester or prior to conception versus selective testing in suspected cases, which is the strategy followed in Romania. Information about prevention measures provided during each prenatal visit could reduce rates of seroconversion. Clinicians should be aware of the most frequent symptoms and signs. They should also perform a follow-up of negative women with regular immunologic tests to detect subsequent seroconversion and primary infection during pregnancy, a strategy implemented in some hospitals.

Antenatal prevention strategies of congenital toxoplasmosis depend on gestational age and consist of spyramicine before 18 weeks gestation, followed by pyrimethamine-sulfadiazine-folinic acid in cases of maternal seroconversion even after 14 weeks of gestation to reduce transplacental transmission. Monitoring the ultrasound signs suggestive of fetal infection and prenatal diagnostic test by amniocentesis are important tools to detect toxoplasmosis in pregnancy followed by postnatal surveillance of the infant.

\section{Acknowledgement}

All authors equally contributed to this article.

Conflict of interest: none declared Financial support: none declared

\section{REFERENCES}

1. De Paschale M, Agrappi C, Manco MT, Cerulli T, Clerici P. Implementation of Screening for Toxoplasma gondii Infection in Pregnancy. J Clin Med Res. 2010;2(3):112-116.

2. Petersen E, Mandelbrot L. Toxoplasmosis and pregnancy. Available at www.uptodate.com.
3. Cytomegalovirus, Parvovirus B19,Varicella Zoster, and Toxoplasmosis in Pregnancy. ACOG Practice Bulletin, No 151, 2015 Jun. www.acog.org.

4. Montoya JG. Laboratory diagnosis of Toxoplasma gondii infection and toxoplasmosis, J Infect Dis. 2002;185(Suppl 1):S73-82. 
5. Peyron P, L'ollivier C, Mandelbrot L, Wallon M, Piarroux R, Kieffer F, Hadjadj E, Paris L, Garcia-Meric P. Maternal and Congenital Toxoplasmosis: Diagnosis and Treatment Recommendations of a French Multidisciplinary Working Group. Pathogens. 2019;8(1):24.

6. Maldonado Al, Read SJ, Committee on Infectious Diseases. Diagnosis, Treatment, and Prevention of Congenital Toxoplasmosis in the United States. Pediatrics. 2017;139(2):e20163860.

7. Stray-Pedersen B. Toxoplasmosis in pregnancy. Baillieres Clin Obstet Gynaecol. 1993;7(1):107-137.

8. Dunn D, Wallon M, Peyron F, Petersen E, Peckham C, Gilbert R. Mother-to-child transmission of toxoplasmosis: risk estimates for clinical counselling. Lancet. 1999;353(9167):1829-33.

9. Wallon M, Garweg JG, Abrahamowicz M, Cornu C, Vinault S, Quantin C, Bonithon-Kopp C, Picot S, Peyron F, Binquet C. Ophthalmic Outcomes of Congenital Toxoplasmosis Followed Until Adolescence. Pediatrics. 2014;133(3):e601-e608.

10. Lange E, Thyrian JR, Wetzka S, Flessa S, Hoffmann W, Zygmunt M, Fusch C, Lode HN, Heckmann M. The impact of socioeconomic factors on the efficiency of voluntary toxoplasmosis screening during pregnancy: a populationbased study. Lange et al. BMC Pregnancy and Childbirth 2016;16:197.

11. Bénard A, Petersen E, Salamon R, Chêne G, Gilbert R, Salmi LR. Survey of European programmes for the epidemiological surveillance of congenital toxoplasmosis. Eurosurveillance 2008;13(15):10.

12. Antenatal care for uncomplicated pregnancies www.nice.org.uk/ guidance/cg62 4 February 2019.

13. Cornu C, Bissery A, Malbos C, Garwig R, Cocherel C, Ecochard R, Peyron F, Wallon M. Factors affecting the adherence to an antenatal screening programme: an experience with toxoplasmosis screening in France. Eurosurveillance 2009;14(9):05.

14. Prusa AR, Kasper DC, Olischar M, Husslein P, Pollak A, Hayde M. Evaluation of serological prenatal screening to detect Toxoplasma gondii infections in Austria. Neonatology 2013;103(1):27-34.

15. Pleyer U, Gross U, Schlüter D, Wilking H, Seeber F. Toxoplasmosis in Germany. Epidemiology, Diagnosis, Risk factors, and Treatment. Dtsch Arztebl Int. 2019; 116(25):435-444.

16. Society for Maternal-Fetal Medicine. Fifteen Things Physicians and Patients Should Question www.choosingwisely.org.

17. Demar M, Hommel D, Djossou F, Peneau C, Boukhari R, Louvel D, Bourbigot AM, Nasser V, Ajzenberg D, Darde ML, Carme B. Acute toxoplasmoses in immunocompetent patients hospitalized in an intensive care unit in French Guyana. Clin Microbiol Infect. 2012; 18(7):E221.

18. McCabe RE, Brooks RG, Dorfman RF, Remington JS. Clinical spectrum in 107 cases of toxoplasmic lymphadenopathy. Rev Infect Dis. 1987;9(4):754.

19. Montoya JG, Remington JS. Management of Toxoplasma gondii infection during pregnancy. Clin Infect Dis. 2008;47(4):554-66.

20. Terra de Oliveira Azevedo C, do Brasil PE, Guida L, Lopes Moreira ME. Performance of Polymerase Chain Reaction Analysis of the Amniotic Fluid of Pregnant Women for Diagnosis of Congenital Toxoplasmosis: A Systematic Reviewand Meta-Analysis. PLoS One. 2016;11(4):e0149938.

21. Gay-Andrieu F, Marty P, Pialat J, Sournies G, Drier de Laforte T, Peyron F. Fetal toxoplasmosis and negative amniocentesis:
Necessity of an ultrasound follow-up. Prenat Diagn. 2003; 23(7):558-60.

22. Filisetti D, Cocquerelle V, Pfaff A, Villard O, Candolfi E. Placental testing for Toxoplasma gondii is not useful to diagnose congenital toxoplasmosis. Pediatr Infect Dis J. 2010;29(7):665-7.

23. Wallon M, Kieffer F, Huissoud C, Peyron F. Cesarean delivery or induction of labor does not prevent vertical transmission of toxoplasmosis in late pregnancy. Int J Gynaecol Obstet. 2015; 129(2):176-7.

24. Robert-Gangneux F, Murat JB, Fricker-Hidalgo $\mathrm{H}$, Brenier-Pinchart MP, Gangneux J P, Pelloux H. The placenta: A main role in congenital toxoplasmosis? Trends Parasitol. 2011;27(12):530-6.

25. Montoya JG, Liesenfeld O. Toxoplasmosis. Lancet. 2004; 363(9425):1965-76.

26. Red Book 2018-2021 Repost of the Committee on Infectious Diseases 31st Edition Toxoplasma gondii Infections. American Academy of Pediatrics https://redbook.solutions.aap.org/.

27. Remington JS, McLeod R Thulliez P, Desmonts G. Toxoplasmosis. In: Remington JS, Klein J. Infectious diseases of the fetus and newborn infant. 5th edn. WB Saunders, Philadelphia 2001:918-1041.

28. Dhombres F, Friszer S, Maurice P, Gonzales M, Kieffer F, Garel C, Jouannic JM. Prognosis of Fetal Parenchymal Cerebral Lesions without Ventriculomegaly in Congenital Toxoplasmosis. Infection Fetal Diagn Ther. 2017;41(1):8-14.

29. Roizen N, Kasza K, Karrison T, Mets M, Noble A G, Boyer K, Swisher C, Meier P, Remington J, Jalbrzikowski J, McLeod R, Kipp M et al. Impact of visual impairment on measures of cognitive function for children with congenital toxoplasmosis: implications for compensatory intervention strategies. Pediatrics 2006; 118(2):e379-90.

30. Mandelbrot L, Kieffer F, Sitta R, Laurichesse-Delmas H, Winer N, Mesnard L, Berrebi A, Le Bouar G et al. TOXOGEST Study Group. Prenatal therapy with pyrimethamine + sulfadiazine vs spiramycin to reduce placental transmission of toxoplasmosis: a multicenter, randomized trial. Am J Obstet Gynecol. 2018;219(4):386.e1-386.e9.

31. Montoya JG. Systematic screening and treatment of toxoplasmosis during pregnancy: is the glass half full or half empty? Am J Obstet Gynecol. 2018;219(4):315-319.

32. Olariu TR, Press C, Talucod J, Olson K, Montoya JG. Congenital toxoplasmosis in the United States: clinical and serologic findings in infants born to mothers treated during pregnancy. Parasite 2019; 26:13.

33. El Bissati K, Levigne P, Lykins J, Adlaoui EB, Barkat A, Berraho A, Laboudi M, El Mansouri B, Ibrahimi A, Rhajaoui M, Quinn F, Murugesan M, Seghrouchni F, Gómez-Marín J E, Peyron F, McLeod R. Global initiative for congenital toxoplasmosis: an observational and international comparative clinical analysis. Emerg Microbes Infect. 2018;7(1):165.

34. Rudin C, Hirsch HH, Spaelti R, Schaedelin S, Klimkait T. Decline of Seroprevalence and Incidence of Congenital Toxoplasmosis Despite Changing Prevention Policy-Three Decades of Cord-blood Screening in North-western Switzerland. Pediatr Infect Dis J. 2018; 37(11):1087-1092. 